\title{
ANÁLISE DO USO DO PENSAMENTO ESTATÍSTICO E DE TÉCNICAS ESTATÍSTICAS NA INDÚSTRIA QUÍMICA DO ESTADO DE SÃO PAULO, BRASIL ${ }^{1}$
}

\section{ANALYSIS OF THE USE OF STATISTICAL THINKING AND STATISTICAL TECHNIQUES IN THE CHEMICAL INDUSTRY OF THE STATE OF SÃO PAULO, BRAZIL}

\author{
Miguel Ángel Aires Borrás* E-mail: maborras@ufscar.br \\ Fabiane Letícia Lizarelli** E-mail: fabiane@dep.ufscar.br \\ José Carlos de Toledo** E-mail: toledo@dep.ufscar.br \\ Manoel Fernando Martins** E-mail: manoel@dep.ufscar.br \\ *Universidade Federal de São Carlos (UFSCar), Sorocaba, SP \\ **Universidade Federal de São Carlos (UFSCar), São Carlos, SP
}

\begin{abstract}
Resumo: As abordagens para a gestão da produção e melhoria são baseadas em tomadas de decisão baseadas em fatos e auxiliadas pelo Pensamento Estatístico (PE) e pelo uso de Técnicas Estatísticas (TE). A pesquisa descrita neste artigo buscou identificar o grau de implementação do PE e TE e, ainda, avalia o seu impacto nos processos de controle e melhoria e a satisfação com essa implementação nas empresas do setor químico localizadas no estado de São Paulo, Brasil. Aplicou-se questionários estruturados e foram realizadas entrevistas em profundidade com especialistas para obtenção de dados e informações. A amostra conseguida é composta por 30 empresas de médio e grande portes. Como principais resultados deste estudo está a percepção de que na maior parte das empresas amostradas há um baixo grau de uso de PE e TE, evidenciando oportunidades para o incremento do desempenho dos processos a partir da aplicação da abordagem estatística e que empresas que não aplicam sistematicamente o PE e as TE têm percebido a necessidade de fazê-lo para potencializar seu desempenho produtivo.
\end{abstract}

Palavras-chave: Pensamento Estatístico. Técnicas Estatísticas. Aplicação. Efeitos. Indústria Química.

Abstract: Approaches to production management and improvement are based on fact-based decisionmaking aided by Statistical Thinking and Statistical Techniques. The research depicted in this article identifies the degree of implementation of statistical thinking and statistical techniques and evaluates the impact on processes control and improvement and satisfaction with this application in chemical companies located in the State of São Paulo, Brazil. The application of a questionnaire and the realization of in-depth interviews with experts were the research techniques applied in that research. The achieved sample is composed of 30 medium and large size companies. As a main result this study figures out that those companies have a low degree of use of statistical thinking and techniques, demonstrating opportunities to improve process performance with statistical approach whereas companies whose do not apply systematically the statistical thinking and techniques have perceived the necessity of do it to improve their productive performance.

Keywords: Statistical Thinking. Statistical Techniques. Application. Effects. Chemical Industry.

\footnotetext{
${ }^{1}$ Este artigo é trabalho inédito, resultado de pesquisa financiada pela Fundação de Amparo à Pesquisa do Estado de São Paulo (FAPESP).
} 


\section{INTRODUÇÃO}

As diversas abordagens difundidas e adotadas para melhoria da produção têm como uma de suas bases científicas a tomada de decisões baseada em fatos e dados, supondo-se que sejam conduzidas com o suporte de princípios e técnicas estatísticas.

Entretanto, a difusão e aplicação desses princípios e técnicas enfrentam limitações e dificuldades para uso rotineiro nas decisões empresariais (PFANNKUCH e WILD, 2004; MAKRYMICHALOS et al., 2005).

Algumas possíveis explicações para essa situação seriam: preconceitos existentes quanto à dificuldade para compreensão e uso desses princípios e técnicas, presentes nos diversos níveis hierárquicos de uma organização; formação deficiente e tardia em Estatística, principalmente de engenheiros e administradores; a não compreensão de que não basta o ensino direto de técnicas estatísticas, se essa formação não for precedida pelo conhecimento e valorização do chamado Pensamento Estatístico ou PE (AHMED e HASSAN, 2003; DOES et al., 1997; ELG et al., 2008; GRIGG e WALLS, 2007).

Pensar estatisticamente significa compreender que a variabilidade, presente em todos os processos e atividades laborais, quando não desejada, precisa ser conhecida e controlada com o apoio de Técnicas Estatísticas (TE), além da adoção de uma visão que refute o determinismo e valorize o comportamento aleatório e a natural variabilidade dos processos (SNEE, 1990; MAKRYMICHALOS et al. 2005; HOERL e SNEE, 2012).

$\mathrm{O}$ uso do PE e das TE tem se ampliado devido à difusão de programas de melhoria, os quais incorporaram em seus princípios o controle estatístico de processo para a análise e solução de problemas (BOX e WOODALL, 2012; GHOSH, 2013; LIM et al., 2014; RAMESH e RAVI, 2013).

Alguns programas para gestão e melhoria da qualidade, como o Kaizen, o TQC, o Seis Sigma ou o Controle Estatístico da Qualidade, pressupõem o uso do PE e das TE e teriam contribuído para uma maior aplicação dessas técnicas (BELEKOUKIAS et al., 2014; GHOSH, 2013; Goh, 2011; HOERL e SNEE, 2010b; KUMAR et al., 2011; MONTGOMERY, 2010; PARK et al., 2017; RAY et al.,2013; SNEE, 1990).

Embora as técnicas estatísticas sejam amplamente discutidas na literatura (ANTONY e BAÑUELAS, 2002; ANTONY e DESAI, 2009; BAÑUELAS et al., 2005; 
GIJO e ANTONY, 2014; WANG e CHEN, 2012) e haja autores que discutam o conceito de Pensamento Estatístico (MACKRYMICHALOS et al., 2005; HOERL e SNEE, 2010b), ainda são poucos os estudos que relacionam as TE e o PE na obtenção de benefícios nas empresas de manufatura e, ainda, existe uma lacuna em determinar como o PE pode ser operacionalizado nesse contexto, especialmente em países em desenvolvimento como o Brasil.

A pesquisa aqui descrita buscou identificar o grau de implementação do PE e TE e, ainda, avalia o seu impacto nos processos de controle e melhoria e a satisfação com essa implementação nas empresas do setor químico localizadas no estado de São Paulo, Brasil. A pesquisa de campo foi conduzida por meio de um levantamento survey, aplicando-se um questionário estruturado em 118 empresas, obtendo-se a resposta de 30 empresas de médio e grande portes. O levantamento limitou-se a empresas de portes médio ou grande, por se considerar um ambiente mais provável para se observar o uso desses princípios e técnicas.

\section{REFERENCIAL TEÓRICO}

\subsection{A relação entre Pensamento Estatístico e Técnicas Estatísticas}

Chance (2002) procura sintetizar a definição de PE, não somente a partir das definições de Wild e Pfannkuck (1999) e Snee (1999), mas também baseada nas definições e observações de Moore (1990), Snee (1990), Cobb (1992), Mallows (1998), Moore (1999) e Smith (1999), evidenciando que o diferencial do pensamento estatístico poderia ser visto como a habilidade de ver o processo como um todo, incluindo o "porquê" para compreender a relação e significado da variação nos processos, tendo a habilidade de explorar os dados além do que é prescrito nos documentos e gerar novas questões além daquelas levantadas pelo principal investigador.

Makrymichalos et al. (2005) e Hoerl e Snee (2012), definem PE como uma filosofia de aprendizado e ação baseada nos seguintes princípios: todo trabalho ocorre num sistema constituído de processos interconectados, a variação existe em todos os

processos e entender e reduzir a variação são elementos-chave para se alcançar o sucesso. 
Para Snee (1999) o comportamento dos processos varia e essa variação gera dados e esses dados são tratados por ferramentas estatísticas para auxiliarem na tomada de decisão. Operacionalmente, o PE pode ser desdobrado em princípios como: medição e coleta de dados sobre os processos, resolução de problema baseada em dados e fatos, busca da melhoria de todo o processo e não de apenas uma operação, reconhecimento e busca da redução da variação nos processos, e análise das possíveis fontes de variação em máquinas, pessoas, materiais, métodos, medições e ambiente (SNEE, 1990; WILD e PFANNKUCH, 1999 e SNEE, 1999). Wild e Pfannkuch (1999) propõe um modelo teórico de quatro dimensões para utilizar o Pensamento Estatístico (PE) em aplicações práticas:

- o ciclo investigativo: baseado no ciclo PDCA, busca definir e compreender o problema e o contexto real do problema com identificação de variáveis e construção de hipóteses sobre suas causas e como o sistema reagiria a mudanças de comportamento de suas variáveis;

- os tipos de pensamento que o tomador de decisão pode assumir para resolver o problema: Wild e Pfannkuch (1999) os chamam de "genéricos", como o pensamento estratégico, e aqueles inerentes ao pensamento estatístico;

- o ciclo interrogativo: onde a busca e refinamento de dados e informações leva à filtragem de ações para resolver os problemas identificados no primeiro ciclo;

- as características pessoais dos envolvidos em tomadas de decisão: consiste em questionar o porquê e como os planos de ação definidos no terceiro ciclo podem ser aplicados e como afetam ou podem afetar o sistema estudado.

Goldman (2005), analisando as origens e o desenvolvimento das iniciativas em qualidade da indústria norte-americana, sustenta que o PE é a base de todos os programas da qualidade, tendo como principal propósito separar as causas comuns de variação das causas especiais, para que ambas possam ser controladas e o desempenho do processo melhorado, sendo que variabilidade existirá independentemente da disponibilidade ou não de dados para estudá-la, enquanto que dados são coletados para melhor entender a variabilidade (SNEE, 1990). 
Para Santos e Martins (2008), o PE consiste numa fundamentação básica para melhoria efetiva dos processos, por possibilitar uma visão mais holística e realista destes, uma vez que com o exame mais pormenorizado dos processos obtém-se um melhor entendimento sobre suas fontes de variação que precisam ser atacadas e possibilitar melhoria do desempenho do processo estudado e para Britz et al. (2000), o PE deve anteceder o uso dos métodos e técnicas estatísticas, posto que o primeiro é conceitual, universalmente aplicável, e as TE tem como requisito principal o conhecimento e a compreensão desse conceito.

O entendimento e prática do PE no nível estratégico da organização daria maior impacto e racionalidade às ações do que propriamente o uso dos métodos estatísticos (HARE et al., 1995; HOERL e SNEE, 2012), uma vez que nos procedimentos de redução de variabilidade dos processos o PE desempenha função fundamental na medida em que estimula a utilização de princípios e técnicas estatísticas pelos níveis tático e operacional (SNEE, 1990; HOERL e SNEE, 2012).

A partir da utilização do PE, a compreensão e a redução dos efeitos das fontes de variação sobre os resultados dos processos podem ser conseguidos com a aplicação de métodos e técnicas estatísticas nas atividades de planejamento, controle e melhoria da qualidade (MAKRYMICHALOS et al., 2005). Os autores Evans e Lindsay (2005) organizam esses métodos e técnicas em três classes: estatística descritiva: métodos e técnicas para a coleta eficiente de conjuntos de dados e sua adequada organização e descrição; inferência estatística: processo de inferir conclusões sobre características desconhecidas de uma população a partir da identificação e caracterização de dados; estatística preditiva: métodos e técnicas para desenhar ou predizer futuros com cenários baseados em dados.

\subsection{Aspectos do uso do Pensamento Estatístico e Técnicas Estatísticas nas organizações}

O Pensamento Estatístico (PE) se faz importante para o estabelecimento de uma abordagem sistêmica para a medição, compreensão e melhoria do desempenho organizacional rumo à excelência em processos e negócios (DRANSFIELD, FISHER, VOGEL, 1999). Porém, apesar da visível importância do uso de PE para a redução e controle da variação dos processos (PRASHAR, 2017), observam-se dificuldades na 
adoção e uso do PE por parte das organizações como, por exemplo, a baixa capacitação de funcionários em estatística e a falta de treinamento para o uso de técnicas e pacotes estatísticos (MAKRYMICHALOS et al., 2005).

Para Coleman (2013), os profissionais da área da qualidade deveriam aderir à evolução do PE e dominar as disciplinas relacionadas à análise de dados e resolução de problemas. A Estatística é praticada principalmente em nível operacional, sendo pouco utilizada nas tomadas de decisão gerenciais (BJERKE e HERSLETH, 2001; SINCLAIR e SADLER, 2004; MAKRYMICHALOS, 2005).

Algumas dificuldades para aplicação do PE e para uso das técnicas estatísticas (TE) são: falta de suporte e envolvimento da alta direção para promover a aplicação do PE e TE (AHMED e HASSAN, 2003; GRIGG e WALLS, 2007; FIRKA, 2011); ausência de conhecimento estatístico adequado pelos gerentes e engenheiros de manufatura (AHMED e HASSAN, 2003; MAKRYMICHALOS et al., 2005; MONTGOMERY, 2010; COLEMAN, 2013); a crença de que a utilização das TE não é suficiente para entregar as melhorias organizacionais, sendo vistas como iniciativas de curto prazo (SINCLAIR e SADLER, 2004; MAKRYMICHALOS et al., 2005; FIRKA, 2011). Alguns autores indicam ações para potencializar o papel e o impacto do PE nas organizações (Quadro 01).

Quadro 01 - Ações para promover maior aplicação do Pensamento Estatístico.

\begin{tabular}{|l|c|}
\hline \multicolumn{1}{|c|}{$\begin{array}{c}\text { Alternativas para impulsionar o uso do } \\
\text { Pensamento Estatístico }\end{array}$} & Autores \\
\hline $\begin{array}{l}\text { Focar o ensino da Estatística no PE em } \\
\text { oposição à ênfase nas técnicas, } \\
\text { contemplando também métodos de análise e } \\
\text { solução de problemas e disciplinas } \\
\text { multidisciplinares. }\end{array}$ & $\begin{array}{c}\text { Brown e Kass (2009), Hoerl e Snee (2010a), } \\
\text { Montgomery (2016) }\end{array}$ \\
\hline $\begin{array}{l}\text { Legitimar a Engenharia Estatística como uma } \\
\text { disciplina acadêmica }\end{array}$ & Hoerl e Snee (2010a) \\
\hline $\begin{array}{l}\text { Estimular o uso do PE, por meio da } \\
\text { Engenharia Estatística, nas organizações. }\end{array}$ & Hoerl e Snee (2010a) \\
\hline $\begin{array}{l}\text { Estimular a participação de estatísticos nas } \\
\text { tomadas de decisões estratégicas das } \\
\text { organizações. }\end{array}$ & Hoerl e Snee (2010a), Mast e Does (2010), \\
\hline
\end{tabular}

Fonte: elaborado pelos autores. 
De acordo com Anderson-Cook et al. (2012), com a evolução do PE e da chamada Engenharia Estatística (EE), tem-se a descoberta de novas ideias e estratégias que proporcionarão soluções rápidas para problemas complexos, considerando-se que o PE deve incluir mais aspectos do pensamento analítico, fazendo melhor uso das análises de dados e estando mais atento a relações de causa e efeito e à redução de desperdício (HOERL e SNEE, 2009; Coleman, 2013).

\section{PROCEDIMENTOS METODOLÓGICOS}

\subsection{Objeto de Estudo}

A importância da indústria química brasileira é notória. De acordo com a Associação Brasileira da Indústria Química ou ABIQUIM (2017a), os segmentos que compõem as atividades da indústria química são contemplados nas divisões 20 e 21 da CNAE 2.0, ou seja, a indústria química no Brasil trabalha com produtos que se dividem, basicamente, em dois grandes blocos: os de uso industrial (Produtos Inorgânicos, Produtos Orgânicos, Resinas e Elastômeros, Produtos e Preparados Químicos Diversos) e os produtos químicos de uso final (Produtos Farmacêuticos, Higiene Pessoal, Perfumaria, Cosméticos, Adubos, Fertilizantes, Sabões, Detergentes e Produtos de Limpeza, Defensivos Agrícolas, Tintas, Esmaltes e Vernizes), ABIQUIM (2017b) e ABIQUIM (2017a).

O objeto de estudo desta pesquisa foi composto pelas empresas fabricantes de produtos químicos de uso industrial. O faturamento do grupo formado por essas empresas representou US $\$ 54,9$ bilhões ou $48,4 \%$ do faturamento total estimado de US\$ 113,5 bilhões da indústria química brasileira em 2016, (ABIQUIM, 2017b).

A limitação geográfica do estudo confinando-se nas fronteiras do estado de São Paulo se deu ao fato de que das 976 plantas fabris de produtos químicos de uso industrial no Brasil, $57,18 \%$ ou 558 plantas se localizam nessa unidade federativa, (ABIQUIM, 2017a).

\subsection{Medição de variáveis}

Baseado em conceitos identificados em revisão bibliográfica, elaborou-se um 
questionário estruturado em quatro partes. A primeira parte contém questões relacionadas à caracterização geral da empresa (porte, setor industrial em que atua, importância estratégica da qualidade, difusão/treinamento/aplicação da PE e TE, razões para o uso não sistematizado de PE e TE, sistemas de certificação da qualidade, e programas de melhoria).

A segunda parte relaciona-se à presença e uso de conceitos do PE. A terceira parte questiona a aplicação de técnicas estatísticas básicas, intermediárias e avançadas. A última parte do questionário traz questões para identificar os benefícios percebidos (objetivos e subjetivos) gerados a partir do uso do PE e TE. As partes 2 a 4 contém sentenças com alternativas que devem ser respondidas usando uma escala de Likert de 1 a 5.

Realizou-se uma análise preliminar da validação interna do questionário com o cálculo do Alfa de Cronbach através do programa Statistica 8.0, a partir dos 30 primeiros respondentes e obtendo-se um valor de 0,9680 para o questionário em geral e para nenhuma questão obteve-se um Alfa abaixo de 0,9666. O valor Alfa para da amostra final de 243 questionários, calculado ao final do survey, foi de 0,97344.

\subsection{Procedimentos de Coleta de Dados}

Após definição do objeto de estudo e identificação da população iniciou-se o envio e aplicação de questionário semiestruturado previamente testado. A coleta das respostas ocorreram ao longo de 9 meses. Do total de 557 plantas fabris de produtos químicos de uso industrial no estado de São Paulo, foi possível identificar e confirmar contatos para envio dos questionários em 118 fábricas, de médio ou grande porte, ou $21,5 \%$ da população, priorizando-se as plantas de maior representatividade econômica, considerando-se número de funcionários e faturamento bruto estimado.

Dos 118 questionários enviados, retornaram 36 respondidos por gerentes da qualidade de empresas de médio e grande portes. Porém, devido a padrões de resposta que se mostraram pouco confiáveis, foram considerados 30 questionários como válidos, ou seja, uma taxa de resposta de aproximadamente $25 \%$ da população acessada. 


\subsection{Procedimentos de Análise de Dados}

Realizou-se uma primeira versão da análise descritiva dos dados da pesquisa survey, seguida de análise multivariada por meio da identificação de agrupamentos de perfis de empresas (análise de cluster) e de análise de associação desses agrupamentos com variáveis como: Importância da Qualidade na estratégia competitiva da empresa; Existência de programas e ações de treinamento em PE/TE; Certificações de Sistemas de Gestão; Programas da Qualidade.

A técnica de agrupamento hierárquico utilizada foi o Método de Ward, o qual não permite que uma empresa altere seu grupo conforme a formação dos agrupamentos e após a definição do número de clusters foi verificada a necessidade de ajuste pelo Método Não-Hierárquico K-means, o qual permite que o indivíduo, no caso a empresa de bens de capital, mude de cluster de acordo com a formação dos grupos (JOHNSON e WICHERN, 2007; GALLARDO, 2011). Salienta-se que são apresentadas apenas as associações aprovadas, ou seja, com $p<0,05$ no teste de Fisher.

\section{RESULTADOS}

\subsection{Perfil da Amostra}

A amostra foi constituída por 18 (60\%) plantas industriais de grande porte (acima de 500 funcionários) e 12 (40\%) de médio porte (entre 100 e 500 funcionários).

Quanto à visão sobre a importância da Qualidade na estratégia competitiva das empresas, observou-se que $87 \%$ (26) das empresas consideram alto esse grau de importância, 10\% (3) consideram médio e 3\% (1) consideram baixo.

$A$ respeito de certificações da qualidade observou-se maior presença das certificações NBR ISO NBR 9001, NBR ISO 14001, BPF/APPCC e NBR ISO TS 16949 em, respectivamente, 18, 11, 4 e 3 empresas da amostra, sendo que algumas possuem mais de uma dessas certificações.

Na Tabela 01 mostra-se um panorama sobre a implantação de Programas da Qualidade nas empresas da amostra. 
Nota-se a elevada parcela de empresas que não fazem uso dos programas como Eventos Kaizen (63\%), Lean Sigma (70\%), TPM (53\%), TQM (63\%), Seis Sigma (40\%) e Lean Manufacturing (50\%) apesar da declarada alta importância da Qualidade (87\% da amostra) na gestão estratégica e na competitividade das empresas.

Ao se analisar a ausência desses Programas observa-se oportunidades de difusão e consolidação dos mesmos nas empresas da amostra, o que poderia resultar na evolução do uso do PE e de TE nessas empresas.

Tabela 01 - Tempo de implantação de Programas da Qualidade nas empresas da amostra.

\begin{tabular}{lcccc}
\hline Programas da Qualidade & $\begin{array}{c}\text { Não } \\
\text { Possui }\end{array}$ & De 0 a 5 anos & De 5 a 10 Anos & Mais de $\mathbf{1 0}$ anos \\
\hline $\begin{array}{l}\text { Iniciativas de Melhoria no } \\
\text { Dia a Dia }\end{array}$ & $10 \%$ & $40 \%$ & $27 \%$ & $23 \%$ \\
$\begin{array}{l}\text { Programa Caixa de } \\
\text { Sugestões }\end{array}$ & $30 \%$ & $43 \%$ & $17 \%$ & $10 \%$ \\
Eventos kaizen & $63 \%$ & $17 \%$ & $10 \%$ & $10 \%$ \\
Seis Sigma & $40 \%$ & $43 \%$ & $7 \%$ & $10 \%$ \\
Lean Manufacturing & $50 \%$ & $33 \%$ & $7 \%$ & $10 \%$ \\
Lean Sigma & $70 \%$ & $20 \%$ & $0 \%$ & $10 \%$ \\
TPM & $53 \%$ & $23 \%$ & $17 \%$ & $7 \%$ \\
TQM & $63 \%$ & $27 \%$ & $10 \%$ & $0 \%$ \\
$5 S$ & $10 \%$ & $53 \%$ & $20 \%$ & $23 \%$ \\
\hline
\end{tabular}

Fonte: pesquisa de campo

Os Programas mais presentes/consolidados na amostra são Iniciativas de Melhoria no Dia a Dia, Caixa de Sugestões e 5S, que intrinsecamente usam menos conhecimentos estatísticos.

Esperava-se um número maior de empresas com certificações, principalmente com NBR ISO 9001 (60\% da amostra) e NBR ISO 14001 (37\%), principalmente desta última, uma vez que trata-se do setor químico, com potencial para impactos ambientes dos produtos e processos, e por serem fornecedores de insumos industriais. O mesmo era esperado para o uso de programas da qualidade.

Esse baixo uso pode se dar pela presença na amostra de empresas de porte médio ( $40 \%$ da amostra) e provavelmente com atuação em mercados que não necessariamente exigem essas certificações, ou por alguma contingência desse setor não captada por essa pesquisa. 
Já em relação aos programas da qualidade, as iniciativas de implantação devem partir da conscientização e determinação interna dos gestores sobre a importância das melhorias para a competitividade.

Faz-se importante ressaltar o contraste entre a relativa baixa presença de certificações da qualidade e de programas de melhoria com o dado que indica uma alta importância atribuída à Qualidade pelas empresas da amostras (87\%).

Em relação ao uso de softwares de gestão, aproximadamente $62 \%$ das empresas usam algum software da família ERP - Enterprise Resource Planning para gestão integrada e $8 \%$ utiliza algum tipo de software de PLM - Product Life-cycle Management. Para tratamento e análise estatística de dados, cerca da metade das empresas (49\%) usa o software Minitab, enquanto 38\% não usa nenhum software específico para métodos estatísticos e as demais empresas se distribuem pelo uso de outros softwares.

\subsection{Presença e aplicação do Pensamento Estatístico e Técnicas Estatísticas}

A Figura 01 apresenta uma síntese dos dados sobre presença de programas e ações específicas para capacitação em PE e TE do pessoal nas empresas, bem como a tendência de investimento nesse tipo de ação.

Figura 01 - Presença de treinamento e capacitação em PE e TE nas empresas

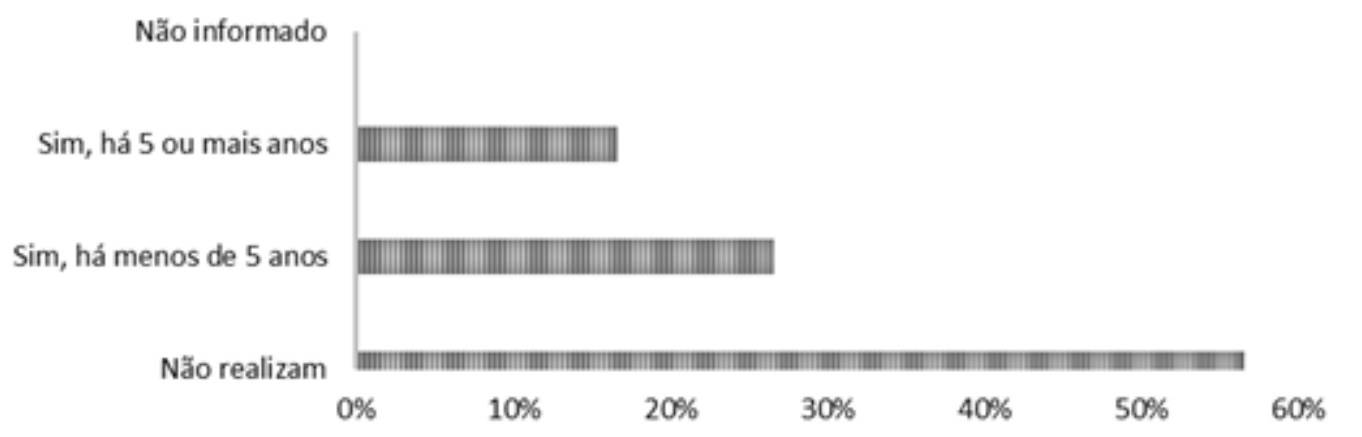

Fonte: pesquisa de campo

Percebe-se que mais da metade (57\%) das empresas não tem nenhum programa de ações focado ou que inclua treinamento e difusão do PE a seus funcionários. Mas, em $43 \%$ amostra esse treinamento já existe, ainda que em sua maioria seja recente, com menos de 5 anos. 
Por outro lado, analisando a Figura 02 , constata-se que $80 \%$ das empresas não têm aumentado ou têm aumentado pouco o investimento em capacitação de seus funcionários em PE e TE.

Para a identificação da presença dos princípios do PE foram levantados dados a partir de 13 questões relacionadas a percepções sobre a variabilidade dos processos e a importância atribuída ao tratamento dos dados e aos sistemas de medição.

Figura 02 - Investimento em treinamento e capacitação em PE e TE nas empresas

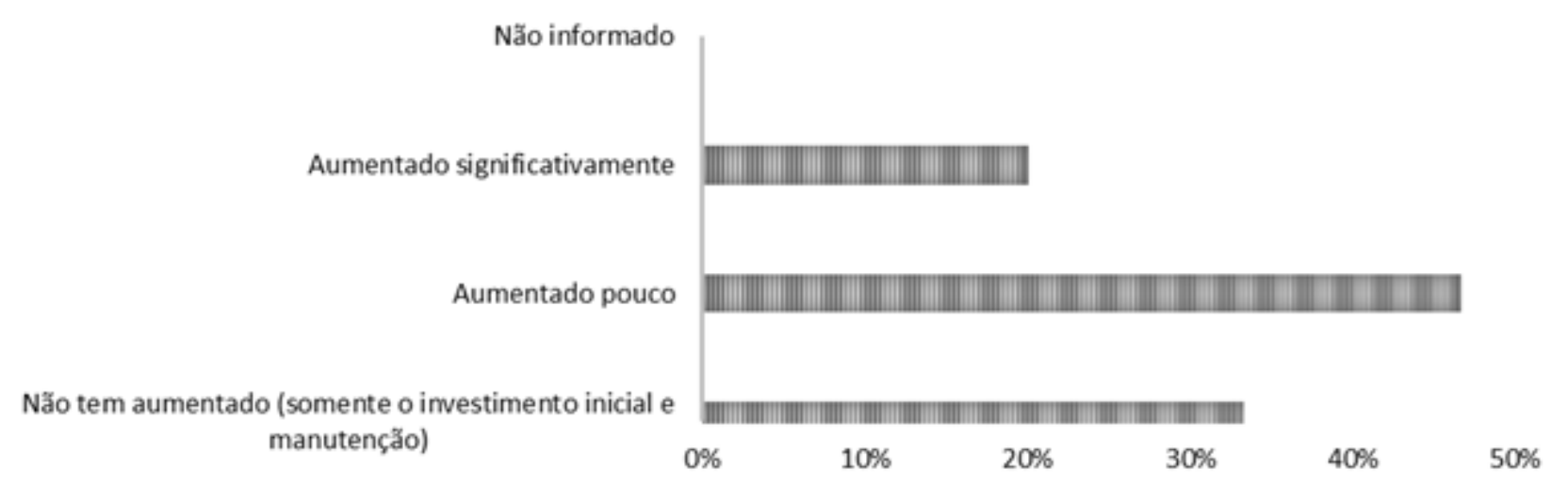

Fonte: pesquisa de campo.

Observou-se que em 53\% das empresas (Tabela 02) não há treinamento continuado sobre PE (Questão 1) porém, 20\% das empresas afirmaram ter elevada frequência desse treinamento e para $40 \%$ a alta administração apoia a implantação do PE, o que revela um certo grau de incentivo ao ensino dos conceitos estatísticos nessas organizações.

Ainda analisando a Tabela 02, percebe-se que $50 \%$ da amostra considera que o uso de PE nunca ou raramente são disseminados e estimulados. Considera-se que a visão de processo está consolidada no nível do pessoal de supervisão e gerencia, mas pouco no pessoal de nível operacional.

O conceito de variabilidade do processo está bem difundido nas empresas (com $73 \%$ das respostas nas alternativas algumas vezes/frequentemente/sempre). Para $84 \%$ das empresas a supervisão e gerência se envolvem e estimulam a coleta e análise de dados. 
Tabela 02 - Resultados sobre a aplicação e difusão de conceitos do Pensamento Estatístico

\begin{tabular}{|c|c|c|c|}
\hline Itens Abordados sobre PE & $\begin{array}{l}\text { Nunca ou } \\
\text { Raramente }\end{array}$ & $\begin{array}{l}\text { Algumas } \\
\text { vezes }\end{array}$ & $\begin{array}{l}\text { Frequentemente } \\
\text { ou Sempre }\end{array}$ \\
\hline $\begin{array}{l}1 \text { - Colaboradores da manufatura } \\
\text { recebem treinamento continuado } \\
\text { sobre PE. }\end{array}$ & $53 \%$ & $27 \%$ & $20 \%$ \\
\hline $\begin{array}{l}2 \text { - Alta administração apoia a } \\
\text { implantação do PE. }\end{array}$ & $30 \%$ & $30 \%$ & $40 \%$ \\
\hline $\begin{array}{l}3 \text { - Uso dos princípios estatísticos } \\
\text { são disseminados e estimulados. }\end{array}$ & $50 \%$ & $33 \%$ & $17 \%$ \\
\hline $\begin{array}{l}4 \text { - O processo de manufatura } \\
\text { principal da unidade está sob } \\
\text { controle estatístico, incorrendo } \\
\text { somente causas comuns. }\end{array}$ & $33 \%$ & $40 \%$ & $27 \%$ \\
\hline $\begin{array}{l}5 \text { - Visão de processo consolidada } \\
\text { no nível de supervisão e gerência. }\end{array}$ & $10 \%$ & $43 \%$ & $47 \%$ \\
\hline $\begin{array}{l}6 \text { - Visão de processo consolidada } \\
\text { no nível de operadores da } \\
\text { manufatura. }\end{array}$ & $40 \%$ & $30 \%$ & $30 \%$ \\
\hline $\begin{array}{l}7 \text { - Conceito de variabilidade do } \\
\text { processo difundido na organização } \\
\text { (entendimento das causas comuns e } \\
\text { causas especiais). }\end{array}$ & $27 \%$ & $23 \%$ & $50 \%$ \\
\hline $\begin{array}{l}8 \text { - Difusão da importância de se } \\
\text { entender e analisar um processo } \\
\text { pela análise quantitativa da } \\
\text { variação. }\end{array}$ & $30 \%$ & $30 \%$ & $40 \%$ \\
\hline $\begin{array}{l}9 \text { - Supervisão e gerência estimulam } \\
\text { e se envolvem na coleta e análise } \\
\text { de dados }\end{array}$ & $17 \%$ & $47 \%$ & $37 \%$ \\
\hline $\begin{array}{l}10 \text { - Difusão da importância da } \\
\text { confiabilidade nos sistemas de } \\
\text { medição }\end{array}$ & $40 \%$ & $47 \%$ & $13 \%$ \\
\hline $\begin{array}{l}11 \text { - Utilização de análise de dados } \\
\text { para suportar a tomada de decisão. }\end{array}$ & $43 \%$ & $17 \%$ & $40 \%$ \\
\hline $\begin{array}{l}12 \text { - Utilização de análise de causa e } \\
\text { efeito baseado em dados dos } \\
\text { processos }\end{array}$ & $27 \%$ & $37 \%$ & $37 \%$ \\
\hline $\begin{array}{l}13 \text { - Difusão do conceito e da } \\
\text { importância da redução da } \\
\text { variabilidade na manufatura. }\end{array}$ & $17 \%$ & $40 \%$ & $43 \%$ \\
\hline
\end{tabular}

Fonte: pesquisa de campo

Em 83\% das empresas há difusão do conceito e da importância da redução da variabilidade na manufatura, pelo menos algumas vezes (40\%) ou 
frequentemente/sempre (43\%). Essas observações apontam que, ao menos nos níveis de supervisão e gerencia, os conceitos e práticas do PE estão no mínimo medianamente implantados nas empresas da amostra.

Em 83\% da amostra, há a difusão do conceito de que a redução da variação é fundamental para o desempenho da manufatura e em $73 \%$ das empresas também há o conceito de variabilidade difundido pela organização.

O aparente apoio da alta administração e a maior incorporação dos conceitos de PE nos níveis de supervisão e gerencia é pouco operacionalizado na prática, pois $50 \%$ das empresas consideram que nunca ou raramente o uso efetivo de princípios estatísticos é estimulado e disseminado (Tabela 02).

Ressalta-se que a visão de processo é mais consolidada no nível de gerencia e supervisão do que de operadores. A difusão da importância da confiabilidade dos sistemas de medição é pouco valorizada na amostra. Isso é frequentemente ou sempre presente em apenas 13\% da amostra (Questão 10).

Embora 37\% das empresas declare realizar análises de causa e efeito com base em dados do processo (Questão 12), 43\% declare ter bem difundido a visão sobre a importância de redução da variabilidade dos processos (Questão 13) e 37\% declare que o nível de Supervisão e Gerência estimula e se envolve na coleta e análise de dados (Questão 9), a preocupação com a qualidade e confiabilidade dos dados utilizados e dos sistemas de medição que os geram ainda é pouco presente.

Verifica-se uma inconsistência conceitual a ser superada, pois as análises mencionadas como sendo realizadas e estimuladas e utilizada frequentemente por supervisores e gerentes, dependem da qualidade dos dados utilizados e resultantes de sistemas de medições.

\subsection{O uso de Técnicas Estatísticas pelas empresas}

Aproximadamente $50 \%$ das empresas declaram fazer uso sistemático de Técnicas Estatísticas (TE).

A Tabela 03 apresenta a frequência de uso de recursos que se considera como evidencias de estimulo e suporte a esse uso, tais como facilitadores, disponibilização de exemplos claros de aplicações e de profissionais com experiência em TE nas atividades e projetos de melhoria. 
Tabela 03 - Uso de facilitadores, exemplos e pessoas com experiência em TE

\begin{tabular}{lccc}
\hline & $\begin{array}{c}\text { Uso de } \\
\text { facilitador } \\
\text { para } \\
\text { treinamento } \\
\text { em TE }\end{array}$ & $\begin{array}{c}\text { Exemplo de } \\
\text { aplicações do uso } \\
\text { de TE } \\
\text { apresentados de } \\
\text { forma clara }\end{array}$ & $\begin{array}{c}\text { Alocação de } \\
\text { pessoas com } \\
\text { experiência em TE } \\
\text { em novos projetos } \\
\text { para aplicação do } \\
\text { conhecimento }\end{array}$ \\
\hline Nunca/Raramente & $63 \%$ & $57 \%$ & $50 \%$ \\
Algumas Vezes & $20 \%$ & $27 \%$ & $27 \%$ \\
Frequentemente/Sempre & $17 \%$ & $17 \%$ & $23 \%$
\end{tabular}

Fonte: pesquisa de campo

De acordo com os dados da Tabela 03, há um baixo ou inexistente uso de facilitadores, disponibilização de exemplos claros de aplicação e alocação de pessoas com experiência em TE em novos projetos de melhoria. Esses dados podem indicar um uso relativamente pouco significativo de TE, principalmente nos níveis operacionais na manufatura.

Esses recursos facilitadores são menos críticos nos níveis de supervisão e gerência que fazem mais uso de PE e TE nessas empresas.

Para avaliar a frequência de aplicação das TE básicas/intermediárias e avançadas, foram analisadas respostas para questões com uma escala de respostas, variando de 1 (nunca) a 5 (sempre), passando pelos valores 2 (raramente), 3 (algumas vezes) e 4 (frequentemente), como mostrado na Tabela 04.

Ao se analisar de forma global os resultados obtidos para técnicas básicas/intermediárias e avançadas, nota-se uma clara diferença na frequência entre o primeiro e o segundo grupo. Enquanto as técnicas básicas e intermediárias são mais amplamente utilizadas, as avançadas possuem um uso significativamente menor.

Tal diferença de utilização de técnicas conforme sua complexidade também foi notada em outros trabalhos sobre aplicação da abordagem estatística, como em Santos e Martins (2010) e em Grigg e Walls (2007), cujas pesquisas abordaram indústrias de alimentos localizadas, respectivamente no estado de São Paulo e no Reino Unido.

Observa-se que as técnicas avançadas não ultrapassaram o percentual de $10 \%$ de uso nas empresas da amostra. A técnica "Análise de Regressão Simples" que foi considerada básica/intermediária na elaboração do instrumento de pesquisa teve frequência de utilização (10\%) semelhante às técnicas avançadas. 
Tabela 04 - Proporção de valores 4 e 5 para as TE consideradas.

\begin{tabular}{|c|c|c|}
\hline & Técnicas Estatísticas & Frequentemente/Sempre \\
\hline \multirow{10}{*}{ 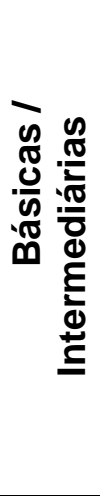 } & Planos de Amostragem & $60 \%$ \\
\hline & Diagrama de Causa e Efeito & $47 \%$ \\
\hline & Folha de Verificação & $47 \%$ \\
\hline & Histograma & $43 \%$ \\
\hline & Diagrama de Pareto & $43 \%$ \\
\hline & $\begin{array}{l}\text { Análise de capacidade processo } \\
\text { (CPK) }\end{array}$ & $40 \%$ \\
\hline & Gráfico de Controle & $37 \%$ \\
\hline & Diagrama de Correlação & $20 \%$ \\
\hline & Princípios de Estratificação & $17 \%$ \\
\hline & Análise de Regressão Simples & $10 \%$ \\
\hline \multirow{7}{*}{ 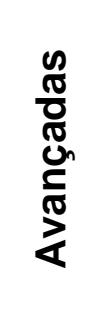 } & Análise do Sistema de Medição (MSA) & $10 \%$ \\
\hline & Analise de Variância - ANOVA & $10 \%$ \\
\hline & Teste de Hipótese & $10 \%$ \\
\hline & Projeto de Experimentos (DOE) & $10 \%$ \\
\hline & Simulação de Fluxos e Processos & $7 \%$ \\
\hline & Análise de Regressão Múltipla & $7 \%$ \\
\hline & Análise Multivariada & $7 \%$ \\
\hline
\end{tabular}

Fonte: pesquisa de campo

Isso indica que essas empresas devem considerar esta técnica como de maior complexidade e/ou não conhecem as possibilidades de uso da mesma. As três técnicas mais amplamente utilizadas são: Planos (Inspeção) de Amostragem (60\% da amostra), Diagrama de Causa-e-Efeito (47\%) e Folha de Verificação (ou Check List, em 47\%).

Com exceção de Planos de Amostragem (Inspeção por Amostragem), pode-se dizer que as outras duas técnicas mais utilizadas são relativamente mais simples e de conteúdo menos estatístico.

As técnicas de gráficos de controle do processo e análise da capacidade do processo (CPk) são usadas por cerca de $40 \%$ da amostra.

A Tabela 05 apresenta a frequência de motivos para o não uso das TE.

Os principais fatores para a não utilização sistemática das TE são: "a falta de conhecimento interno que suporte à aplicação" (33\% das que responderam que não fazem uso sistemático), "a ausência de uma cultura adequada que estimule a aplicação do PE e TE” (67\%) e "aplica-se muito esporadicamente, em função de demandas externas e outras prioridades da empresa" (73\%). 
Tabela 05 - Frequência de justificativas para a não aplicação sistemática das TE

Motivos da não aplicação

Porcentagem em relação às negativas

Aplica-se muito esporadicamente, em função de demandas externas e outras prioridades da empresa

$73 \%$

Falta de uma cultura adequada para aplicação de PE/TE

$67 \%$

Falta de conhecimento interno que suporte a aplicação

sistemática

$33 \%$

De modo geral o nível de formação e de conhecimento

do pessoal não permite essa aplicação

$27 \%$

Tem-se uma visão predominante de que a aplicação de

PE/TE é complexa

$27 \%$

Os gestores veem as TE somente como algo para se

'combater incêndios'

$27 \%$

Os gestores acreditam que as TE só podem ser

utilizadas no chão de fábrica e não são importantes para

questões gerenciais e estratégicas

Não sentimos a necessidade de aplicação

sistemática/rotineira

Não se acredita no potencial da aplicação de PE/TE

Fonte: pesquisa de campo

\subsection{Resultados percebidos com a aplicação de PE e TE}

Para avaliação dos resultados percebidos com aplicação do PE e de TE foram formuladas 15 questões (Tabela 06).

Em relação aos resultados percebidos pela empresa, os melhores resultados são referentes a indicadores mais objetivos tais como a Redução de não conformidades internas na planta industrial (40\%), a Redução de não conformidades identificadas no mercado (taxa de devolução ou reclamações) (43\%), o Aumento no nível de satisfação dos clientes (40\%) e o Aumento na produtividade (47\%), conforme mostrado na Tabela 06 a seguir. 
Tabela 06 - Resultados percebidos pela empresa com a aplicação do PE e de TE

\begin{tabular}{|c|c|c|c|}
\hline Resultado com aplicação do PE e TE & $\begin{array}{l}\text { Nenhum / } \\
\text { Pequeno }\end{array}$ & Moderado & $\begin{array}{c}\text { Grande/ } \\
\text { Muito Grande }\end{array}$ \\
\hline $\begin{array}{l}1 \text { - Redução de não conformidades } \\
\text { internas na planta industrial }\end{array}$ & $37 \%$ & $23 \%$ & $40 \%$ \\
\hline $\begin{array}{l}2 \text { - Redução de não conformidades } \\
\text { identificadas no mercado (taxa de } \\
\text { devolução ou reclamações). }\end{array}$ & $37 \%$ & $20 \%$ & $43 \%$ \\
\hline $\begin{array}{l}3 \text { - Aumento no nível de satisfação dos } \\
\text { clientes. }\end{array}$ & $33 \%$ & $27 \%$ & $40 \%$ \\
\hline $\begin{array}{l}4 \text { - Redução nos custos da não } \\
\text { qualidade. }\end{array}$ & $37 \%$ & $30 \%$ & $33 \%$ \\
\hline $\begin{array}{l}5 \text { - Redução no tempo de ciclo de } \\
\text { produção. }\end{array}$ & $47 \%$ & $17 \%$ & $37 \%$ \\
\hline $\begin{array}{l}6 \text { - Redução nos custos de produção } \\
\text { decorrente do PE e TE }\end{array}$ & $47 \%$ & $23 \%$ & $30 \%$ \\
\hline 7 - Aumento na produtividade & $33 \%$ & $20 \%$ & $47 \%$ \\
\hline $\begin{array}{l}8 \text { - Melhoria nos índices de capacidade } \\
\text { do processo (Cpk). }\end{array}$ & $37 \%$ & $30 \%$ & $33 \%$ \\
\hline $\begin{array}{l}9 \text { - Nível de satisfação dos tomadores de } \\
\text { decisão com o uso do PE. }\end{array}$ & $47 \%$ & $20 \%$ & $33 \%$ \\
\hline $\begin{array}{l}10 \text { - Colaboradores do nível operacional } \\
\text { se sentem motivados para aplicação do } \\
\text { PE e TE. }\end{array}$ & $63 \%$ & $23 \%$ & $13 \%$ \\
\hline $\begin{array}{l}11 \text { - O nível de compreensão dos } \\
\text { processos e da capacidade de resolução } \\
\text { de problemas com o uso do PE e das } \\
\text { TE. }\end{array}$ & $50 \%$ & $27 \%$ & $23 \%$ \\
\hline $\begin{array}{l}12 \text { - Contribuição do PE e TE para } \\
\text { consolidação dos programas de melhoria } \\
\text { (Seis Sigma, Lean, TPM, etc). }\end{array}$ & $53 \%$ & $13 \%$ & $33 \%$ \\
\hline $\begin{array}{l}13 \text { - Redução da variabilidade e melhoria } \\
\text { da estabilidade dos processos. }\end{array}$ & $53 \%$ & $17 \%$ & $30 \%$ \\
\hline $\begin{array}{l}14 \text {-Aumento do comprometimento com } \\
\text { a medição quantitativa da variabilidade } \\
\text { dos processos e suas causas. }\end{array}$ & $43 \%$ & $23 \%$ & $33 \%$ \\
\hline $\begin{array}{l}15 \text { - Contribuição à mudança cultural da } \\
\text { organização em prol da qualidade. }\end{array}$ & $43 \%$ & $20 \%$ & $37 \%$ \\
\hline
\end{tabular}

Fonte: pesquisa de campo.

Como indicadores menos expressivos destacam-se a pouca percepção de resultados como "os funcionários do nível operacional se sentem motivados para aplicar o PE e as TE" (resultado 10: 63\% na soma das alternativas nenhum ou pequeno), "aumento do nível de compreensão do processo e da capacidade de 
resolução de problemas com o uso do PE e das TE" (resultado 11: 50\%) e "contribuição do PE e TE para consolidação dos programas de melhoria (resultado 12: $53 \%$ ) e Redução da variabilidade e melhoria da estabilidade dos processos com o PE e TE (resultado 13: 53\%).

\section{ANÁLISES DE CLUSTER E DE ASSOCIAÇÃO}

Os grupos formados inicialmente, por meio do método de Ward estão apresentados no dendograma da Figura 03, a partir da qual pode-se identificar que 0 melhor agrupamento ocorre ao se considerar três clusters.

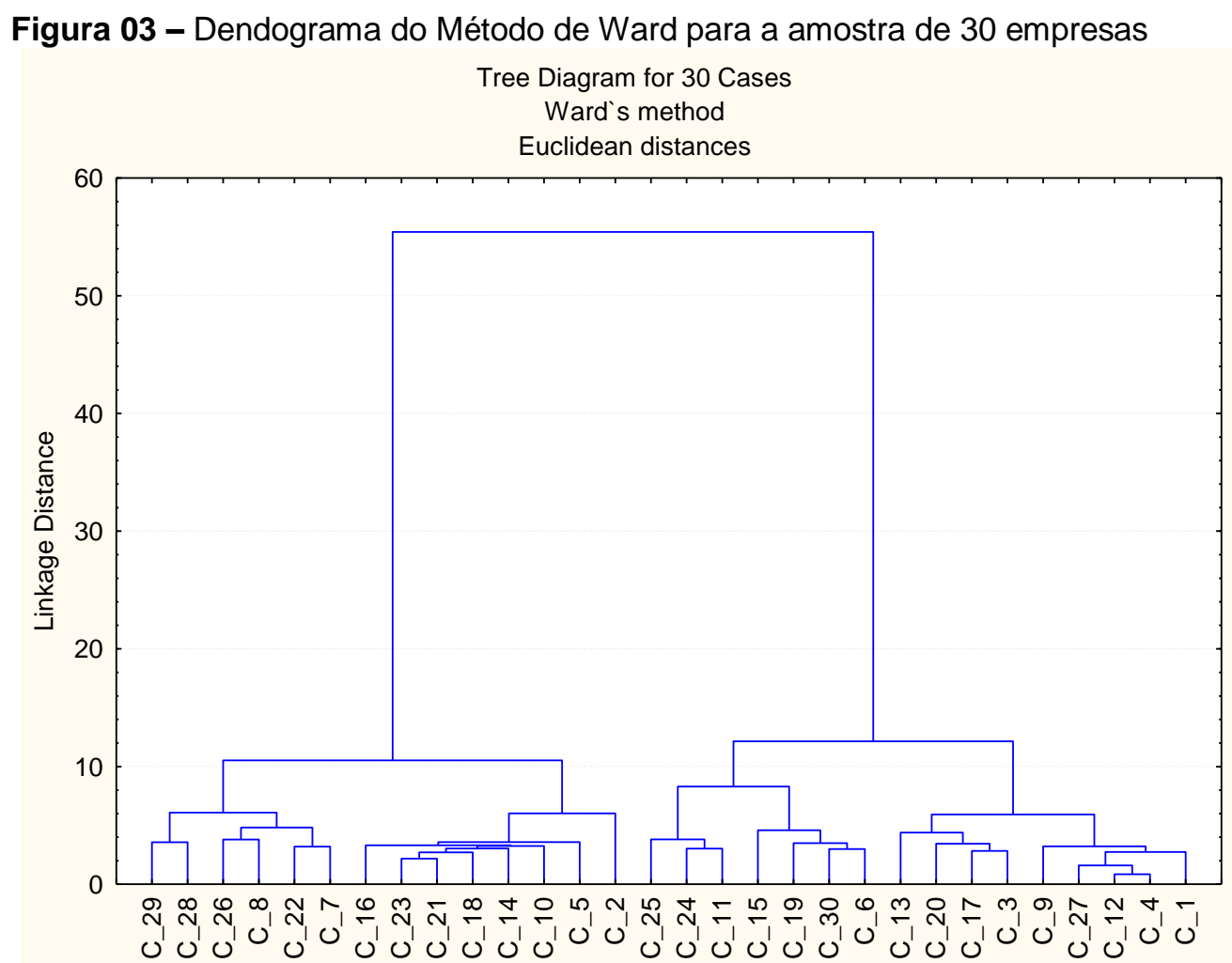

Fonte: pesquisa de campo

As variáveis referentes ao uso de PE e ao uso de TE básicas avançadas foram sintetizadas em uma única variável. Já as variáveis relativas ao desempenho estão apresentadas individualmente nas respostas (RE) 1 a 15.

A partir da análise do dendograma da Figura 03, tem-se o agrupamento das empresas amostradas em três clusters: 
- cluster 1: C_2, C_5, C_8, C_10, C_14, C_16; C_18, C_22, C_23, C_26 e C_29;

- cluster 2: C_1; C_3; C_4; C_12; C_13; C_17; C_20 e C_27;

- cluster 3: C_6; C_7, C_9, C_11, C_15, C_19, C_24, C_25, C_28 e C_30.

Ressalta-se que não se fez necessário um ajuste na distribuição das empresas entre os três clusters definidos pelo método hierárquico, o que pode ser ratificado com o resultado da análise realizada com o Método Não-Hierárquico K-means (Figura 04).

Figura 04 - Gráfico do Método Não-Hierárquico K-means para a amostra de 30 empresas

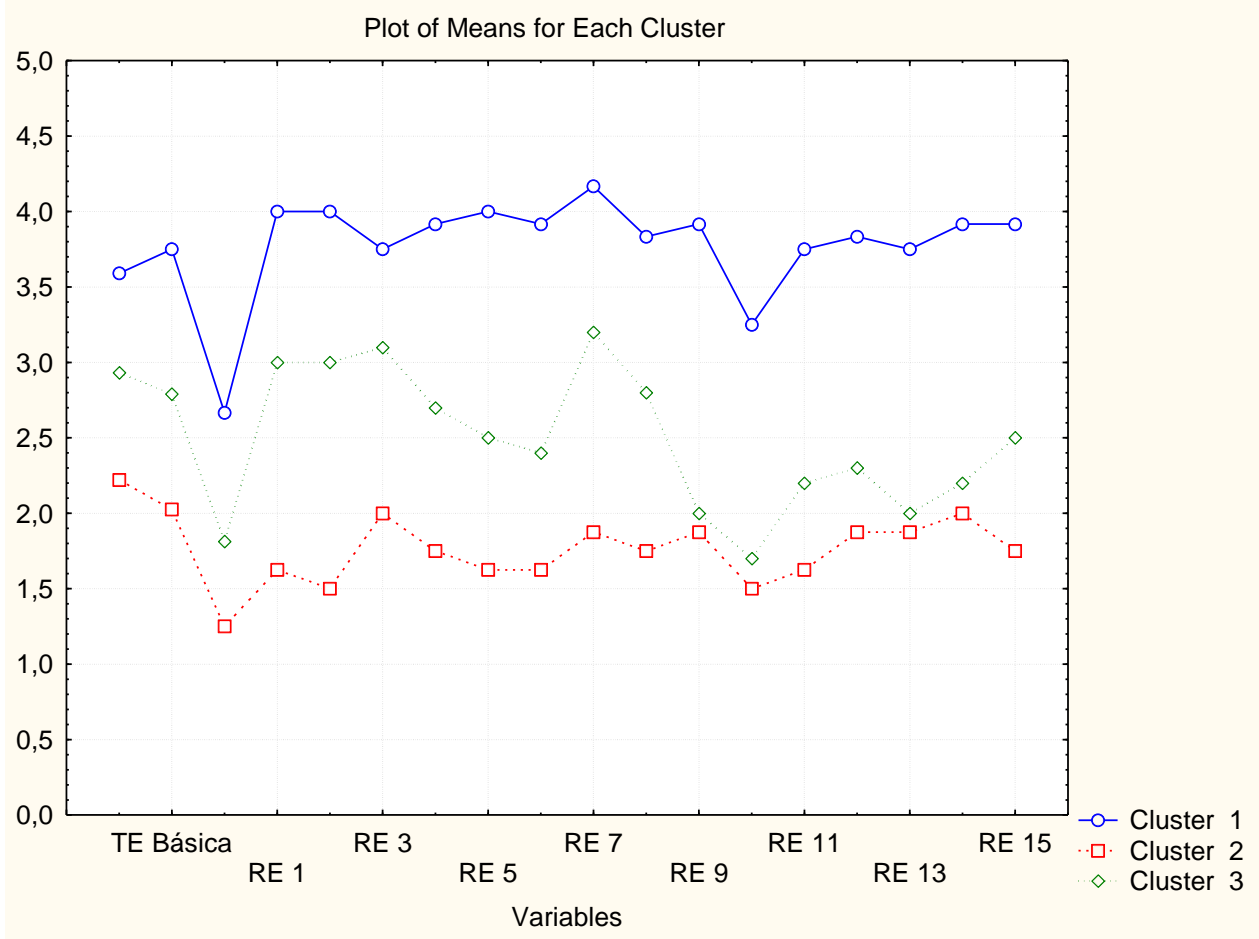

Fonte: pesquisa de campo

Analisando os resultados obtidos, observou-se que o uso de PE no setor químico de produtos de uso industrial no estado de São Paulo, é mais intenso (nos grupos 2 e 3) do que o uso de TE básicas.

Também se constatou que o uso de TE avançadas é bem menos intenso que o de PE e de TE básicas. Entre os Clusters 2 e 3 há pouca diferença nos resultados de percepção mais subjetiva (RE 9 a RE 15). Há uma maior porcentagem de empresas (12 unidades) no Cluster de maior intensidade de uso de PE e TE. 
Para a Análise de Associação entre os clusters e o uso de Programas de Melhoria, considerou-se apenas as associações que apresentaram um valor $p$ inferior a $5 \%$, no caso somente para os programas TPM e TQM (Tabela 07).

Tabela 07 - Análise de Associação para o uso de TPM e TQM.

\begin{tabular}{lcccc}
\hline \multicolumn{2}{c}{ Programa e Resposta sobre o Programa } & Cluster 1 & Cluster $\mathbf{2}$ & Cluster 3 \\
\hline NPa & & 1 & 4 & 8 \\
Poso possui & & 8 & 2 & 2 \\
p-valor & TQM & & 0,00814 & \\
& & Cluster 1 & Cluster 2 & Cluster 3 \\
Não possui & & 1 & 4 & 8 \\
Possui & & 8 & 2 & 2 \\
p-valor & & & 0,00814 & \\
\hline
\end{tabular}

Fonte: elaboração própria

Pela leitura da Tabela 07, verifica-se que das 12 empresas do Cluster 1, 9 responderam sobre Programa TPM, das quais 1 não possui o Programa e 8 possuem. A análise evidencia que possuir o Programa TPM ou o Programa TQM exerceu influência no uso de PE e TE, uma vez que o cluster 1 é o de maior sudo relativo de $P E, T E$ e de melhores resultados percebidos.

A significância na utilização de TPM e TQM nessa amostra do setor. Em relação ao TPM, apenas 1 empresa (11\%) do Cluster 1 não utiliza. Já no Cluster 2, de menor desempenho relativo, 4 empresas (67\%) não possuem TPM.

O Cluster 3, com desempenho intermediário nas variáveis analisadas para a formação dos clusters, possui um comportamento parecido com o do Cluster 2 em relação ao uso do TPM (80\% das empresas não o utiliza).

Evidencia-se a associação entre uso do TPM e as variáveis utilizadas para formação dos Clusters, ocorrendo o mesmo em relação ao TQM, em que $90 \%$ das empresas do Cluster 1 utilizam o TQM, diferentemente dos Clusters 2 e 3, em que respectivamente 33\% e 25\% utilizam esse Programa.

Os Clusters 1 e 3 (com alto e médio uso de PE e TE, respectivamente) possuem mais empresas com programas e ações focados em difusão e treinamento de PE e TE, como pode ser visto na Tabela 08. 
Dentre os fatores para os quais não houve associação com os agrupamentos chamou a atenção que a certificação NBR ISO 9001, presente em $60 \%$ da amostra, não exerceu influência no uso de PE e TE.

Tabela 08 - Análise de Associação para o Difusão e Treinamento do PE e de TE

Programas e ações para difusão, treinamento e aplicação do PE e de TE

Cluster 1 Cluster 2 Cluster 3

\begin{tabular}{lccc}
\hline Não & 4 & 8 & 5 \\
Sim, há menos de 5 anos & 5 & 0 & 3 \\
Sim, há 5 ou mais anos & 3 & 0 & 2 \\
p-valor & & 0,052928 &
\end{tabular}

Fonte: elaboração própria

Possuir a certificação NBR ISO 9001, nessa amostra, não influencia o uso de PE e TE (Tabela 09), o mesmo ocorrendo com o uso do Programa Seis Sigma, neste caso contrariando a expectativa, por ser o programa que prevê estimulo e uso mais intenso de PE e TE.

Tabela 09 - Análise de Associação dos clusters com NBR ISO 9001 e Programa Seis Sigma

NBR ISO 9001

Cluster 1

Cluster 2

Cluster 3

Sim

Não

7

3

3

6

p-valor

Não possui

Programa Seis Sigma

Possui

3

p-valor

1

1

0,814241

Cluster 1 Cluster 2 Cluster 3

$\begin{array}{lll}9 & 3 & 6\end{array}$

Fonte: elaboração própria

\section{CONCLUSÕES}

Percebeu-se que as empresas da amostra ainda não estão totalmente inseridas na filosofia do Pensamento Estatístico (PE), demonstrando que há oportunidades para melhorar o controle e desempenho dos processos por meio da utilização dos princípios do PE e de técnicas estatísticas (TE).

Apesar da maioria das empresas perceberem que compreendem o conceito de variabilidade e entendem que sua redução e controle são necessários para a melhoria 
de processos e obtenção de ganhos de desempenho, apenas uma pequena parcela da amostra aplica o PE, capacita os funcionários em PE e investe no incremento do grau de confiabilidade dos dados coletados, que é básico para o uso adequado do PE e TE.

Com relação ao uso de TE, apesar da maioria das empresas reconhecerem a importância dessas técnicas para a melhoria de seus processos, apenas uma pequena parcela as aplicam sistematicamente. Além disso, quando há uso de TE a maior parte das empresas se concentra na aplicação das técnicas básicas e não inclui, significativamente, pessoas com experiência em uso de TE em novos projetos de melhoria de processos e produtos e nem mesmo faz uso de exemplos e de facilitadores para incrementar o uso do PE e das TE.

As empresas dessa amostra de fabricantes de produtos químicos de uso industrial, apesar de reconhecerem a importância do uso do PE e TE, fazem um uso pouco sistemático de TE e do PE, sendo que menos da metade das empresas capacitam seu pessoal nesses conceitos e técnicas, dado que em $57 \%$ das amostra não há treinamento formal em PE e TE.

As próprias empresas percebem que o uso do PE e TE é limitado pela falta de conhecimento interno e pela falta de uma cultura organizacional adequada voltada para o seu uso rotineiro. Evidencia-se também algumas inconsistências conceituais, como por exemplo a importância atribuída ao conhecimento e controle da variabilidade e a valorização da tomada de decisões de processo baseada e fados e dados, e a perceptível pouca importância atribuída à confiabilidade dos sistemas de medição que geram os dados para análise e decisões sobre produtos e processos.

Conclui-se que são necessárias iniciativas de discussão entre entidades do setor, representantes das empresas, institutos de pesquisa e universidades, para um diagnóstico em conjunto e plano de ações para superar as dificuldades e robustecer o uso de PE e TE nas empresas da indústria química, buscando o melhor controle da variabilidade para o incremento do desempenho operacional.

\section{REFERÊNCIAS}

ABRAHAN, B. Statistics in Business and Industry: implementation. International Statistical Review, v.73, n.2, p.173-176, 2005. https://doi.org/10.1111/j.1751-5823.2005.tb00263.x 
AHMED, S.; HASSAN, M. Survey and case investigations on application of quality management tools and techniques in SMls. International Journal of Quality \& Reliability Management, v. 20, n. 7, p. 795-826, 2003. https://doi.org/10.1108/02656710310491221

ANDERSON-COOK, C.M. et al. Statistical engineering: forming the foundations. Quality Engineering, v. 24, n. 2, p.110-132., 2012. https://doi.org/10.1080/08982112.2012.641150

ANTONY, J.; BAÑUELAS, R. Critical success factors for the successful implementation of Six Sigma projects in organizations. The TQM magazine, v. 14 n. 2, p. 92-99, 2002. https://doi.org/10.1108/09544780210416702

ANTONY, J.; DESAI, D. A. Assessing the status of Six Sigma implementation in the Indian industry: results from an exploratory empirical study. Management Research News, v. 32, n. 5, p. 413-423, 2009. https://doi.org/10.1108/01409170910952921

ASSOCIAÇÃO BRASILEIRA DA INDÚSTRIA QUÍMICA. A Indústria Química Brasileira. São Paulo, 2017. Disponível em:

http://www.abiquim.org.br/includes/pdf/indQuimica/AlndustriaQuimica-Conceitos.pdf. Acesso em: 24/04/2018.

O Desempenho da Indústria Química Brasileira em 2016. São Paulo, 2017. Disponível em: http://www.abiquim.org.br/includes/pdf/indQuimica/livreto-de-dados-2016paginas.pdf. Acesso em: 24 abr. 2018.

BAÑUELAS, R.; ANTONY, J.; BRACE, M. An application of Six Sigma to reduce waste. Quality and Reliability Engineering International, v. 21, n. 6, p. 553-570, 2005. https://doi.org/10.1002/qre.669

BELEKOUKIAS, I.; GARZA-REYES, J. A.; KUMAR, V. (2014), The impact of lean methods and tools on the operational performance of manufacturing organisations, International Journal of Production Research, v. 52, n. 18, p. 5346-5366, 2014. https://doi.org/10.1080/00207543.2014.903348

BJERKE, F.; HERSLETH, M. Introducing statistical thinking to the food industry: facilitating and inhibiting factors. Quality Management Journal, v.8, n.3, p.49-60, 2001. https://doi.org/10.1080/10686967.2001.11918966

BOX, G. E. P.; WOODALL, W. H. Innovation, quality engineering, and Statistics, Quality Engineering, v. 24, n. 1, p. 20-29, 2012. https://doi.org/10.1080/08982112.2012.627003

BRITZ, G. C. et al. Improving performance through statistical thinking. 1. ed. Milwaukee: ASQ Quality Press, 2000. 177 p.

CHANCE, B. L. Components of Statistical Thinking and Implications for Instruction and Assessment. Journal of Statistics Education, v. 10, n. 3, p. 1-14, 2002.

https://doi.org/10.1080/10691898.2002.11910677

COBB, G. Teaching Statistics. In: Heeding the call for change: suggestions for curricular action. In: STEEN, L. A. MAA Math Notes 22. Washington: Mathematical Association of America, p. 3-34, 1992.

COLEMAN, S.Y. Statistical Thinking in the quality movement \pm 25 years. The TQM Journal, v.25, n.6, p.597-605, 2013. https://doi.org/10.1108/TQM-06-2013-0075 
DOES, R.J.M.M.; TRIP, A.; SCHIPPERS, W.A.J. A framework for implementation of statistical process control. International Journal of Quality Science, v. 2, n. 3, p. 181-198, 1997. https://doi.org/10.1108/13598539710170821

DRANSFIELD, S. B.; FISHER, N. I.; VOGEL, N. J. Using statistics and statistical thinking to improve organisational performance. International Statistical Review, v. 67, n. 2, p. 99122, 1999. https://doi.org/10.1111/j.1751-5823.1999.tb00417.x

ELG, M.; OLSSON, J.; DAHLGAARD, J. Implementing statistical process control: an organizational perspective. International Journal of Quality \& Reliability Management, v. 25, n. 6, p. 545-560, 2008. https://doi.org/10.1108/02656710810881872

EVANS, J. R.; LINDSAY, W. M. The management and control of quality. Mason: Thomson South-Western. 6. ed., 2005. 760 p.

FIRKA, D. Statistical, technical and sociological dimensions of design of experiments. The TQM Journal, v. 23, n. 4, p. 435-445, 2011. https://doi.org/10.1108/17542731111139509

GALLARDO, J. Métodos jerárquicos de análisis cluster. Curso de Diplomatura Estadística Teórico Práctico de la Universidad de Granada, 2011. Disponível em: <http://www.ugr.es/ gallardo/pdf/cluster-3.pdf>. Acesso em: 24 abr. 2018.

$\mathrm{GHOSH}, \mathrm{M}$. Lean manufacturing performance in Indian manufacturing plants. Journal of Manufacturing Technology Management, v. 24, n. 1, p. 113-122, 2013. https://doi.org/10.1108/17410381311287517

GIJO, E. V.; ANTONY, J. Reducing patient waiting time in outpatient department using lean six sigma methodology. Quality and Reliability Engineering International, v. 30, n. 8, p. 1481-1491, 2014. https://doi.org/10.1002/qre.1552

GOH, T. N. Raising statistical literacy for manufacturing productivity. International Journal of Quality \& Reliability Management, v. 7, n. 3, p. 27-34, 1990.

https://doi.org/10.1108/02656719010138669

GOLDMAN, H. H. The Origins and Development of Quality Initiatives in American Business. The TQM Magazine, v. 17, n. 3, p. 217-225, 2005. https://doi.org/10.1108/09544780510594180

GRIGG, N.; WALLS, L. Developing statistical thinking for performance improvement in the food industry. International Journal of Quality \& Reliability Management, v. 24, n.4, p. 347-369, 2007. https://doi.org/10.1108/02656710710740536

HARE, L.B. Linking statistical thinking to Six Sigma. International Journal of Six Sigma and Competitive Advantage, v.1, n.4, p.389-402, 2005.

https://doi.org/10.1504/IJSSCA.2005.008505

HARE, L. B. Statistical engineering- principles and examples. Quality Engineering, v.24, n.2, p.153-161, 2012. https://doi.org/10.1080/08982112.2012.641144

HOERL, R. W.; SNEE, R. D. Closing the gap: statistical engineering links statistical thinking, methods and tools. Quality Progress, p. 52-53, 2010.

Statistical Thinking: improving business performance. 2.ed. New Jersey: John Wiley \& Sons, 2012. 514 p. 
JOHNSON, R. A.; WICHERN, D. W. Applied multivariate statistical analysis. 6 ed. Boston: Pearson High Education USA, 2007. 800 p.

KUMAR, M.; ANTONY, J.; TIWARI, M.K. Six Sigma implementation framework for SMEs: a roadmap to manage and sustain the change. International Journal of Production Research, v. 49, n. 18, p. 5449-5467, 2011. https://doi.org/10.1080/00207543.2011.563836

LIM, S. A. H.; ANTONY, J.; ABLIWI, S. Statistical Process Control (SPC) in the food industry: a systematic review and future research agenda. Trends in Food Science \&

Technology, v. 37, n. 2, p. 137-151, 2014. https://doi.org/10.1016/j.tifs.2014.03.010

MAKRYMICHALOS, M. et al. Statistical thinking and its role for industrial engineers and managers in the 21st century. Managerial Auditing Journal, v.20, n.4, p.354-363, 2005. https://doi.org/10.1108/02686900510592043

MALLOWS, C. The zeroth problem. The American Statistician, v. 52, p. 1-9, 1998. https://doi.org/10.1080/00031305.1998.10480528

MONTGOMERY, D. C. A modern framework for achieving enterprise excellence. International Journal of Lean Six Sigma, v.1, n.1, p.56-65, 2010. https://doi.org/10.1108/20401461011033167 2016. $549 p$.

Introdução ao Controle Estatístico da Qualidade. 4. ed. Rio de Janeiro: LTC,

PARK, M.; KIM, J. J. Y.; KWON, K. M.; YU, G. J. Process control and economic cost design for total quality management. Total Quality Management \& Business Excellence, v. 28, n. 7-8, p. 858-878, 2017. https://doi.org/10.1080/14783363.2015.1133243

RAMESH, N.; RAVI, A. TQM tools and techniques in promoting team working culture in the manufacturing organizations. International Journal of Productivity and Quality

Management, v. 12, n. 4, p. 466-479, 2013. https://doi.org/10.1504/IJPQM.2013.056777

RAY, S et al. Measuring Six Sigma project effectiveness using fuzzy approach. Quality and Reliability Engineering International, v. 29, n. 3, p. 417-430; 2013.

https://doi.org/10.1002/qre.1391

SANTOS, A. B.; MARTINS, M. F. Modelo de referência para estruturar o Seis Sigma nas organizações. Gestão \& Produção, v.15, n.1, p.43-56, 2008. https://doi.org/10.1590/S0104$\underline{530 \times 2008000100006}$

Contribuições do Seis Sigma: estudo de caso em multinacionais. Produção, v.20, n.1, p.42-53, 2010. https://doi.org/10.1590/S0103-65132010005000003

SINCLAIR, K. A.; SADLER, B. A. Going beyond SPC: why we need statistical thinking in operations such as carbon plants. In: TOMSETT, A.; JOHNSON, J. (ed) Essential Reading in Light Metals. Wiley: TMS, v.4, 2013. p.365-370. https://doi.org/10.1002/9781118647745.ch48

SMITH, T. M. F. Discussion in response to Wild and Pfannkuch. International Statistical Review, v. 67, n. 3, p. 248-250, 1999. https://doi.org/10.1111/j.1751-5823.1999.tb00443.x

SNEE, R. D. Discussion: "development and use of statistical thinking: a new era". International Statistical Review, v. 67, n. 3, p. 255-258, 1999. https://doi.org/10.1111/i.17515823.1999.tb00446.x 
Getting Better Business Results: using statistical thinking and methods to shape the bottom line. Quality Progress, p.102-106, 1998.

Six Sigma: the evolution of 100 years of business improvement methodology. International Journal of Six Sigma and Competitive Advantage, v. 1, n. 1, p. 4-20, 2004. https://doi.org/10.1504/IJSSCA.2004.005274

Statistical thinking and its contribution to total quality. The American Statistician, v.44, n.2, p.116-121, 1990. https://doi.org/10.2307/2684144

WANG, F. K.; CHEN, K. S. Application of Lean Six Sigma to a panel equipment manufacturer. Total Quality Management \& Business Excellence, v. 23, n. 3-4, p. 417429, 2012. https://doi.org/10.1080/14783363.2011.593876

WILD, C. J.; PFANNKUCH, M. Statistical Thinking in Empirical Enquiry. International Statistical Review, v. 67, n. 3, p. 223-265, 1999. https://doi.org/10.2307/1403699

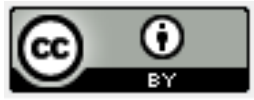

Artigo recebido em: 26/04/2018 e aceito para publicação em: 29/05/2019

DOI: http://dx.doi.org/10.14488/1676-1901.v19i2.3234 